\title{
PROCESS DEVELOPMENT \& MICROSTRUCTURE \& MECHANICAL PROPERTY EVALUATION OF A DUAL MICROSTRUCTURE HEAT TREATED ADVANCED NICKEL DISC ALLOY
}

\author{
R.J. Mitchell ${ }^{1}$, J.A. Lemsky ${ }^{2}$, R. Ramanathan ${ }^{2}$, H.Y. Li ${ }^{3}$, \\ K.M. Perkins ${ }^{4}$, L.D. Connor ${ }^{5}$ \\ ${ }^{1}$ Rolls-Royce plc, PO Box 31, Derby, DE24 8BJ, UK \\ ${ }^{2}$ Ladish Co., Inc., PO Box 8902, Cudahy, WI 53110, USA \\ ${ }^{3}$ Department of Metallurgy and Materials Science, School of Engineering, \\ University of Birmingham, Edgbaston, Birmingham, B15 2TT, UK \\ ${ }^{4}$ Materials Research Centre, School of Engineering, Swansea University, \\ Singleton Park, Swansea, SA2 8PP \\ ${ }^{5}$ Department of Materials Science and Metallurgy, University of Cambridge, \\ Pembroke Street, Cambridge, CB2 3QZ
}

Keywords: RR1000, Dual-Microstructure, Heat Treatment

\begin{abstract}
This paper summarises Rolls-Royce's efforts in developing a production capable and industrially robust process for the production of dual-microstructure components. This has been possible through the use of process modeling, full-scale validation trials, detailed microstructural assessment through a variety of destructive and non-destructive methods and mechanical property evaluation. This paper reports the fundamental underlying relationships between thermal history and microstructural evolution and how these directly influence mechanical properties. This work has enabled a deeper understanding of the opportunities and difficulties faced in the application of this technique for producing dual microstructures, and how the technique can be scaled up from the research laboratory to full production capacity.
\end{abstract}

\section{Introduction}

Today's large civil gas turbine engines rely on chemically complex nickel-base superalloys for turbine discs and other critical rotatives. These alloys are typically produced via powder metallurgy before being formed into shape by isothermal forging. Through appropriate forging and subsequent heat treatment parameters, components can be produced with grain sizes lying in one of three size ranges, fine grain (ASTM 13-10, 4-11 $\mu \mathrm{m})$, midi grain (ASTM 10-7, 11-32 $\mu \mathrm{m}$ ) or coarse grain (ASTM 7-3, 32$125 \mu \mathrm{m})$.

Hot-section gas turbine components, such as turbine discs, experience a large radial thermal and mechanical loading gradient during operation. The bore region of the disc operates at relatively low temperatures and high stresses whilst the rim is subject to less severe mechanical loading at a higher temperature. The rim region requires good creep and fatigue crack growth resistance best achieved using coarse grain microstructures, whereas the bore region requires tensile and fatigue strength optimised by use of fine grain microstructures.

Components produced with a single microstructure throughout inevitably result in a trade-off in design and/or component life. Therefore, it is desirable to produce dual-microstructure components with the grain size optimised at different locations, based upon the likely in-service requirements. Depending upon the starting microstructure, this may manifest itself in either an increased temperature capability, an increase in allowable rotational speed or an increase in component life.

In recent years a number of methods for the production of dualmicrostructure components have been reported [1, 2, 3, 4]. The techniques that have been developed by individual parties are all subtly different in terms of approach and each have their respective merits and limitations. However, all of the methods developed rely upon the same basic principle of maintaining a temperature gradient throughout the component during the solution heat treatment. In fine grain regions, grain growth is restricted by Zener pinning by primary $\gamma^{\prime}$. Solution heat treating a portion of the component above the $\gamma^{\prime}$ solvus removes the primary $\gamma^{\prime}$ and allows grain growth. A uniform coarse grain structure can be produced if stored energy within the grains is minimised through appropriate thermo-mechanical working.

This paper concentrates on Rolls-Royce's efforts in developing an industrially robust, production capable process and illustrates this via detailed results for two turbine disc components that have been produced in the high temperature nickel disc alloy, RR1000 [5]. RR1000 is a third generation, powder processed, $\gamma^{\prime}$ precipitation strengthened nickel-base superalloy the composition of which is shown below in Table 1.

Table 1 Composition of RR1000 in wt \%

\begin{tabular}{|c|c|c|c|c|c|c|c|c|c|c|}
\hline Ni & Cr & Co & Mo & Al & Ti & Ta & Hf & Zr & C & B \\
\hline bal & 15 & 18.5 & 5 & 3 & 3.6 & 2 & 0.5 & 0.06 & 0.027 & 0.015 \\
\hline
\end{tabular}

RR1000 powder was produced via argon gas atomisation and then extruded. Mults were cut from the extruded billet and isothermally forged into various component geometries before being subject to the Dual Microstructure Heat Treatment (DMHT) process.

This paper focuses on two very different turbine disc geometries that have been produced with a dual-microstructure as part of the development activities at Rolls-Royce. The first geometry, A, is a small turbine disc with forged dimensions of $\sim 350 \mathrm{~mm}$ in diameter and a maximum thickness (height) of $\sim 65 \mathrm{~mm}$. The minimum section thickness is $\sim 28 \mathrm{~mm}$. The second geometry, B, is a much larger turbine disc (Figure 1) with forged dimensions of $\sim 675 \mathrm{~mm}$ in diameter and a maximum section thickness (height) of $\sim 250 \mathrm{~mm}$. The minimum section thickness is $\sim 65 \mathrm{~mm}$. 
This paper explores the microstructural and mechanical property characterisation of two dual-microstructure components produced in two different geometries, typical of the range of products produced by Rolls-Royce. The microstructures of both components have been extensively characterised in terms of grain size, grain boundary serration, $\gamma^{\prime}$ size, morphology and volume fraction, and have been reported for component $\mathrm{A}$ in a previous paper by the authors [6].

This paper concentrates on the microstructural and mechanical property evaluation of the larger component, $\mathrm{B}$, and compares and contrasts this with the previously reported work. Results of mechanical property testing are discussed with reference to the thermal history and microstructure.

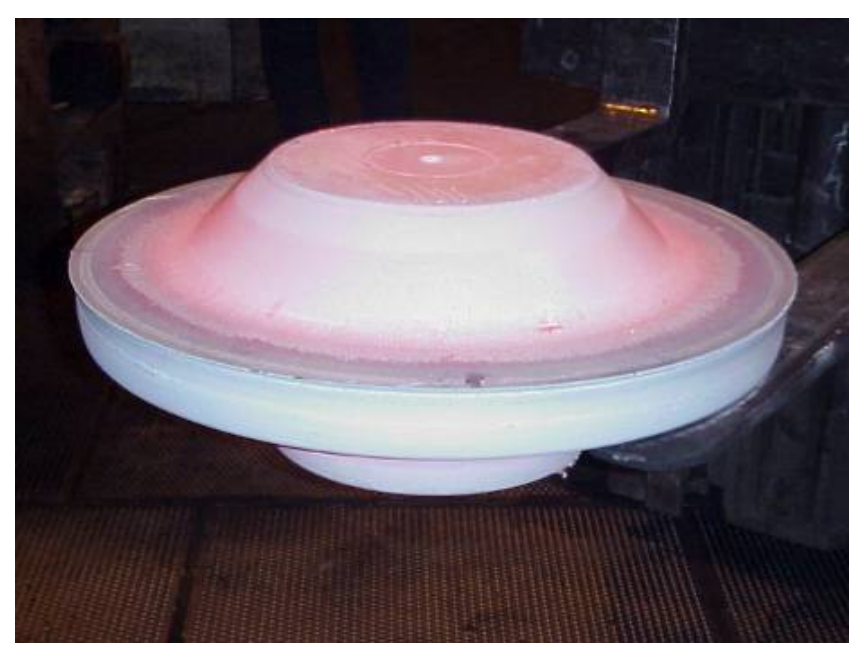

Figure 1 Large High Pressure (HP) turbine disc (B) as forged

\section{Process Development}

The process used in developing an industrially robust dualmicrostructure heat treatment process is summarised below:

- Component design

- Define forging geometry

- Define grain size requirement

- Produce preliminary process models

- Design DMHT apparatus

- Manufacture thermocoupled validation component

- Conduct process model validation trials on thermocoupled component

- Produce validated process model

- Produce first part

The method that has been developed by Rolls-Royce for producing dual-microstructures in brief comprises sets of thermal insulators located at key locations on the component, for example the bore of a turbine disc forging, whilst other areas are left exposed. The assembly is then placed into a standard production furnace at a temperature above the $\gamma^{\prime}$ solvus allowing grain coarsening to occur in the exposed areas. At an appropriate time the component and assembly are removed from the furnace and the two are then separated, allowing the component to be cooled according to standard industrial practices.
This approach requires the use of robust process modeling techniques coupled with careful selection of insulation material and furnace parameters, in addition to geometrical information to define an appropriate heat treatment schedule. In the case of a High-Pressure (HP) turbine disc, this allows grain coarsening in the rim area, whilst the bore temperature is maintained close to the subsolvus solution temperature.

Rolls-Royce specified the desired location of the transition region between grain sizes based upon a number of design and manufacturing considerations. Ladish then produced preliminary process models showing the feasibility of the approach and initial insulation material designs. These initial models were based on well established boundary conditions and thermo-physical property data.

A validation component was then manufactured and thermocouples placed at numerous key locations. The validation trials were performed to acquire actual thermal response data to match to model predictions and to establish a precise heating plan for the RR1000 forgings. Figure 2 depicts the numerous locations chosen for thorough temperature interrogation.

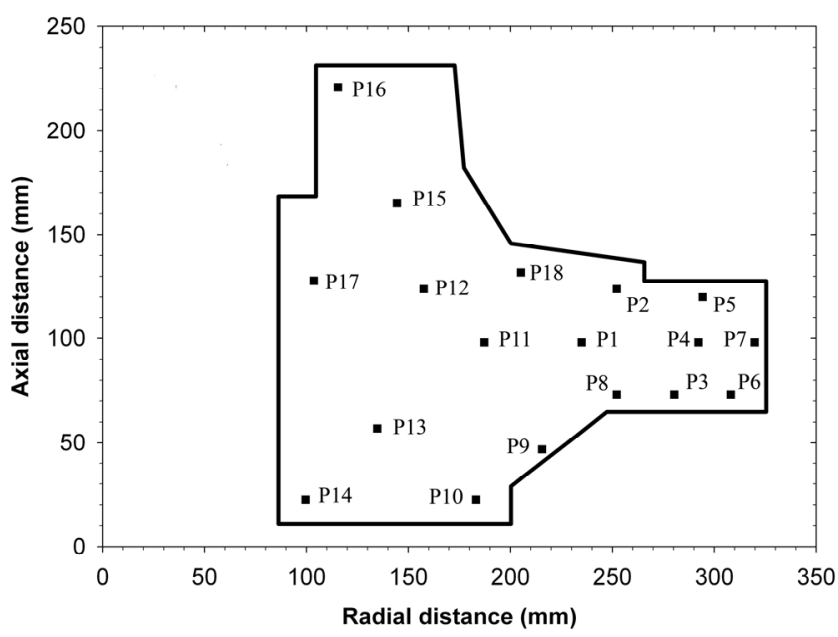

Figure 2 Locations of thermocouples in validation component

Figure 3 below shows the simulated and experimental temperature data, expressed relative to the $\gamma^{\prime}$ solvus for a bore and rim thermocouple during the heat-up part of the cycle. The high fidelity process models developed at Ladish over a number of years resulted in a minimal number of heat treatment iterations being required to validate the models. 


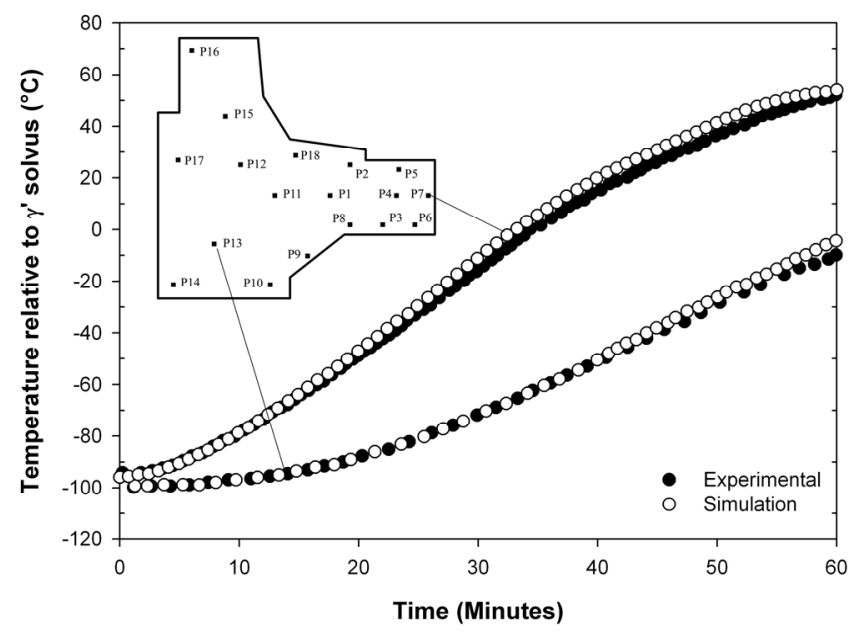

Figure 3 Typical temperature correlation for heat-up part of cycle - simulated versus experimental

The thermocoupled validation discs also serve the purpose of determining the achievable cooling rate in the various locations within the forging. This, when coupled with advanced cooling technology such as the Ladish Supercooler ${ }^{\mathrm{TM}}$ enables cooling rates to be tailored in different locations of the forging to achieve the desired mechanical properties.

Once the thermal process models have been validated then the benefit of the Rolls-Royce developed methodology is that no external monitoring or fixturing is required to successfully produce production parts. These thermal models when coupled with grain growth models allow accurate prediction of final component grain sizes for use in design far in advance of the manufacture of the first part.

Having successfully validated the process model for both heating and cooling events it is then possible to interrogate the model to provide details on a variety of factors that may be of importance in determining the microstructural response to the DMHT heat treatment.

Figure 4 shows the predicted temperature profile for the large HPT disc heat treat shape (B) at the end of the dual microstructure heat treatment step. Knowledge of the $\gamma^{\prime}$ solvus suggests that the transition region is likely to lie in the region where the $\gamma^{\prime}$ solvus temperature is exceeded. However, this takes no account of either the grain growth or primary $\gamma^{\prime}$ dissolution kinetics of the alloy system. The plot shows the instantaneous temperature at the end of the DMHT cycle and confirms that the chosen furnace parameters have resulted in a good temperature gradient from rim to bore. The bore temperature attained is consistent with that of a standard subsolvus solution temperature.

Interrogating the process models further enables a series of variables to be assessed, which can in turn be linked to the microstructure that evolves.

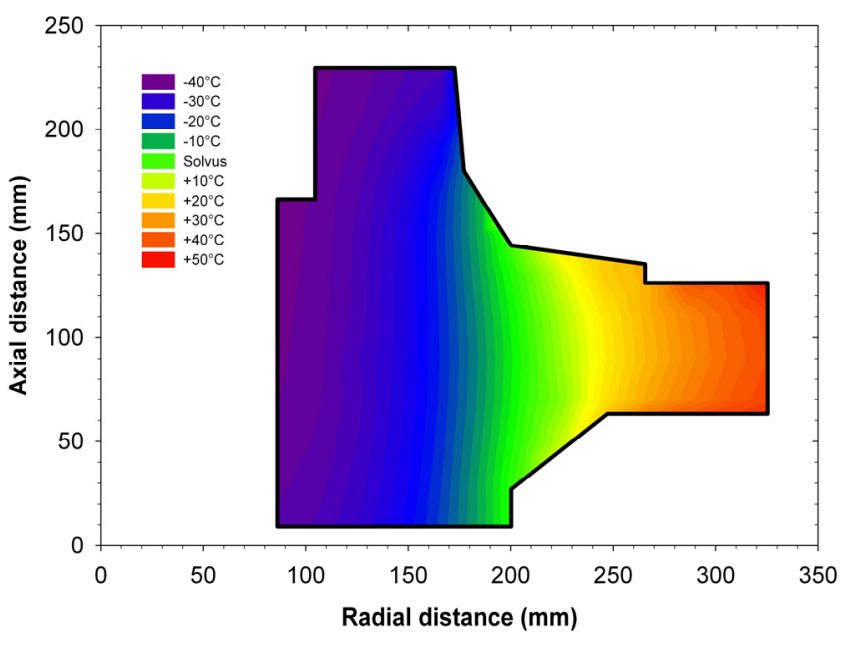

Figure 4 Temperature at the end of the DMHT cycle

Figure 5 shows the time above $\gamma^{\prime}$ solvus that each region of the component witnesses. The bore region, remains below the $\gamma^{\prime}$ solvus at all times and the exposed rim region sees the longest amount of time above the $\gamma^{\prime}$ solvus. The furnace parameters were chosen with knowledge of the grain growth kinetics of RR1000 to ensure that the rim region of the forging was above the $\gamma^{\prime}$ solvus for a sufficient period of time to achieve the desired grain size in the rim region.

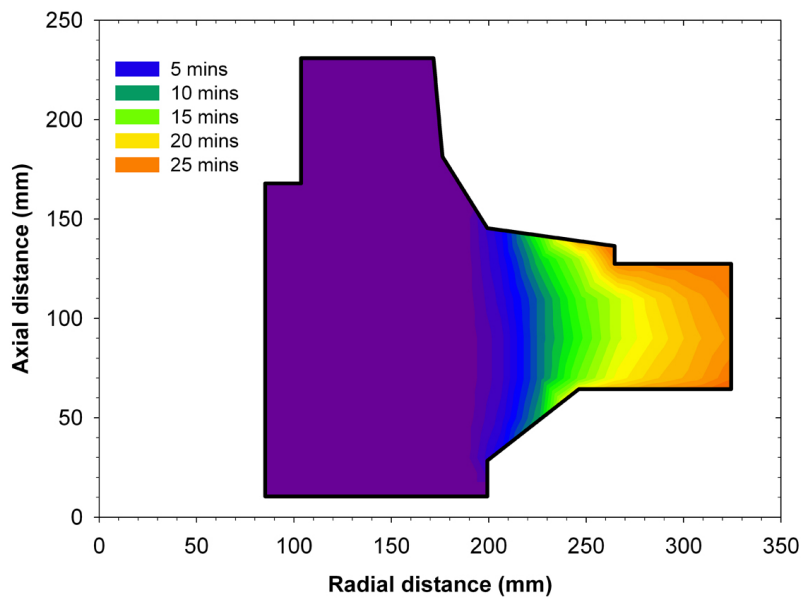

Figure 5 Time above $\gamma^{\prime}$ solvus temperature

Figure 5 shows, as expected that the time gradient within the forging follows the temperature gradient. The majority of the rim region has seen $>20$ minutes above the $\gamma^{\prime}$ solvus, this time decreasing with decreasing radial distance. The presence of the gradual temperature gradient within the component is advantageous as it produces a gradual transition region between grain sizes, the nature of which can be adjusted to suit the design requirements.

In addition to modeling the heat-up part of the DMHT cycle Ladish also modeled and validated cooling rates within the component. Residual stress predictions were also produced and compared to both modeled and measured values produced in dualmicrostructure and uniform structure components. Subsequent to the cooling cycle, the components were subject to the normal ageing heat treatment for RR1000. 


\section{Microstructural Assessment}

\section{Grain Structure and Primary $\gamma^{\prime}$}

This section focuses on the microstructural assessment of a single macro-slice removed from a forging produced in geometry B. The macro-slice was sectioned into one hundred and four individual twenty by twenty millimetre micro-specimens. Each one was then mounted, ground and polished to a high finish using standard metallographic techniques. The micro-specimens were then etched using a modified Kallings solution to reveal grain boundary structure. For analysis of the $\gamma^{\prime}$ precipitates samples were re-polished and etched electrolytically using $10 \%$ phosphoric acid in water at $3-5 \mathrm{~V}$ for $3-5$ seconds. Grain size analyses were performed in accordance with ASTM E112 [7].

Figure 6 shows how the average grain size varies with radial distance in geometry B. In the bore region grain size is a consistent $5-8 \mu \mathrm{m}$ (ASTM 12-11). This increases to a consistent 25-30 $\mu \mathrm{m}$ (ASTM 7.5) in the rim region. The As Large As (ALA) grain size measured in the fine grain region was $13 \mu \mathrm{m}$ (ASTM 9.5) and $105 \mu \mathrm{m}$ (ASTM 3.5) in the coarse grain region. Based on the grain sizes measured the transition region between the two microstructures is around $35 \mathrm{~mm}$ in width.

In addition to measuring the grain size, image analysis was also performed on the primary $\gamma^{\prime}$ precipitates. A piece of software called ImageJ was used to determine primary $\gamma^{\prime}$ volume fraction and precipitate size distributions (PSD's) for each micro-specimen [8]. The software has the capability of determining the presence of precipitates based on their differential contrast to that of the matrix phase. By setting an appropriate threshold level the software effectively counts the number of pixels present as primary $\gamma^{\prime}$ and if they are conjoined assumes they are a single precipitate. In this way outputs of area fraction and a size histogram are obtained for each image analysed. For each of the one hundred and four locations a minimum of one hundred precipitates were measured.

Figure 7 shows how the primary $\gamma^{\prime}$ mean size varies as a function of location. Figure 8 shows the volume fraction information on the same basis. In the bore region of the turbine disc forging it is apparent that the mean primary $\gamma^{\prime}$ size is between $1-2 \mu \mathrm{m}$ and the volume fraction is between $12-16 \%$. Complete dissolution of the primary $\gamma^{\prime}$ has occurred in the coarse grain region as expected.

Reviewing the three plots, Figure 6 - Figure 8 altogether, it is apparent that simply considering grain size as a means of defining the transition region is not appropriate. Figure 8 shows that as radial distance increases the volume fraction of primary $\gamma^{\prime}$ decreases whilst in Figure 7 it is seen that the mean $\gamma^{\prime}$ size decreases at a slower rate. The grain size does not begin to increase until the volume fraction has decreased to a certain level and in-turn the mean average primary $\gamma^{\prime}$ size has also reduced. The results presented seem to suggest that even when the primary $\gamma^{\prime}$ volume fraction is low, grain growth will not occur whilst some primary $\gamma^{\prime}$ resides on the grain boundary, even at a small mean average size. However, it should also be considered that the whole system is experiencing a spatially variant temperature and that the kinetics of $\gamma^{\prime}$ dissolution will vary with the time and temperature experienced by each location in the near/super solvus regions.

Representative macro / micrographs are shown in Figure 9.

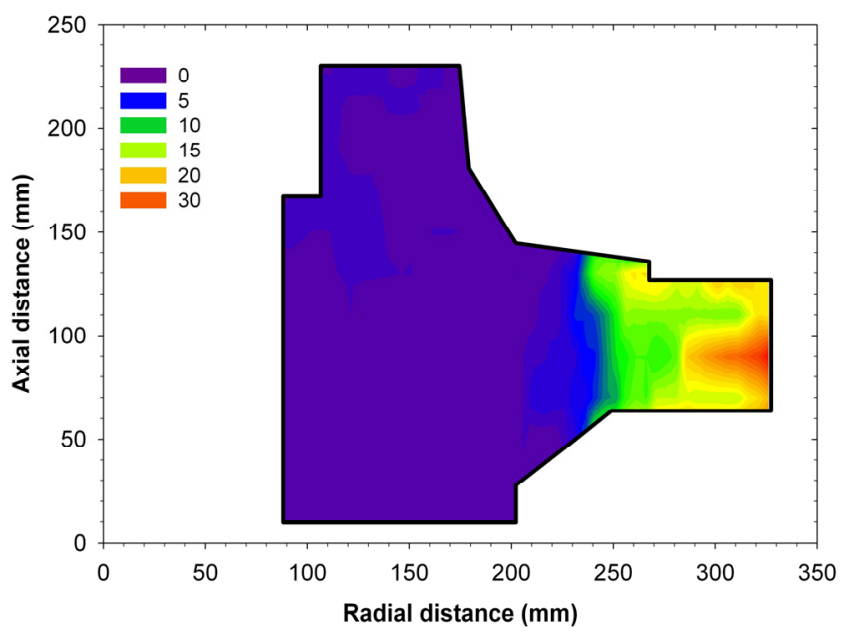

Figure 6 Average grain size $(\mu \mathrm{m})$ in dual-microstructure forging

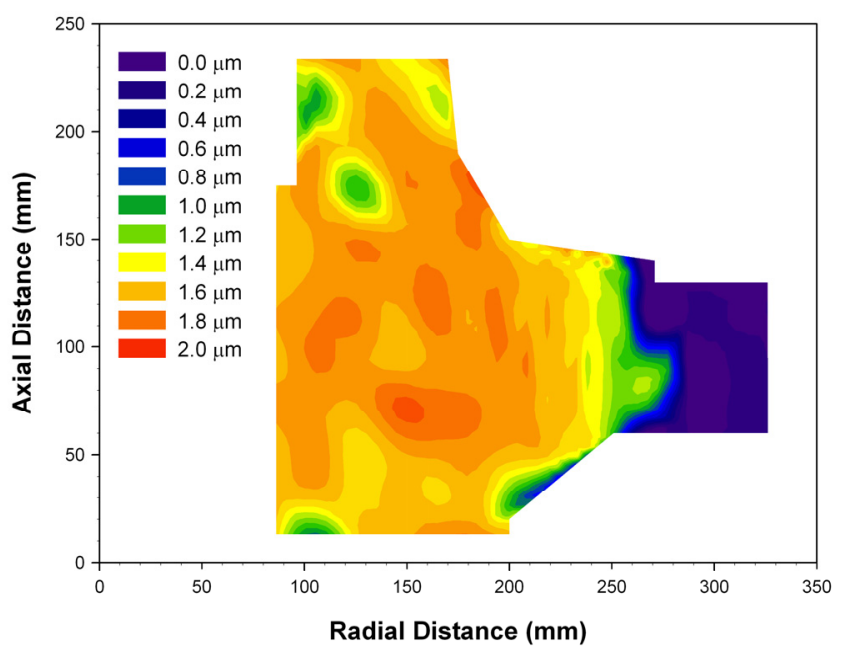

Figure 7 Variation in primary $\gamma^{\prime}$ size with location

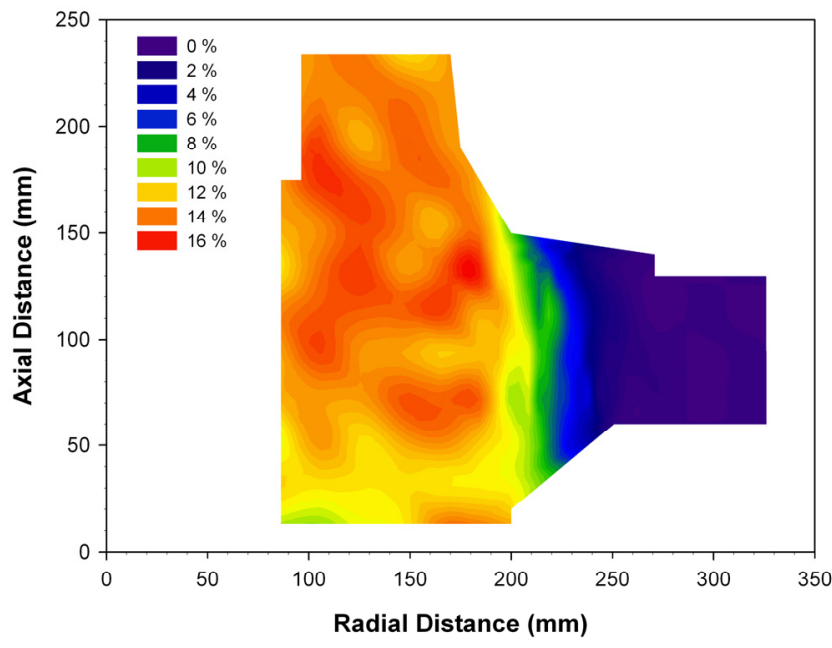

Figure 8 Volume fraction of primary $\gamma^{\prime}$ as a function of location 

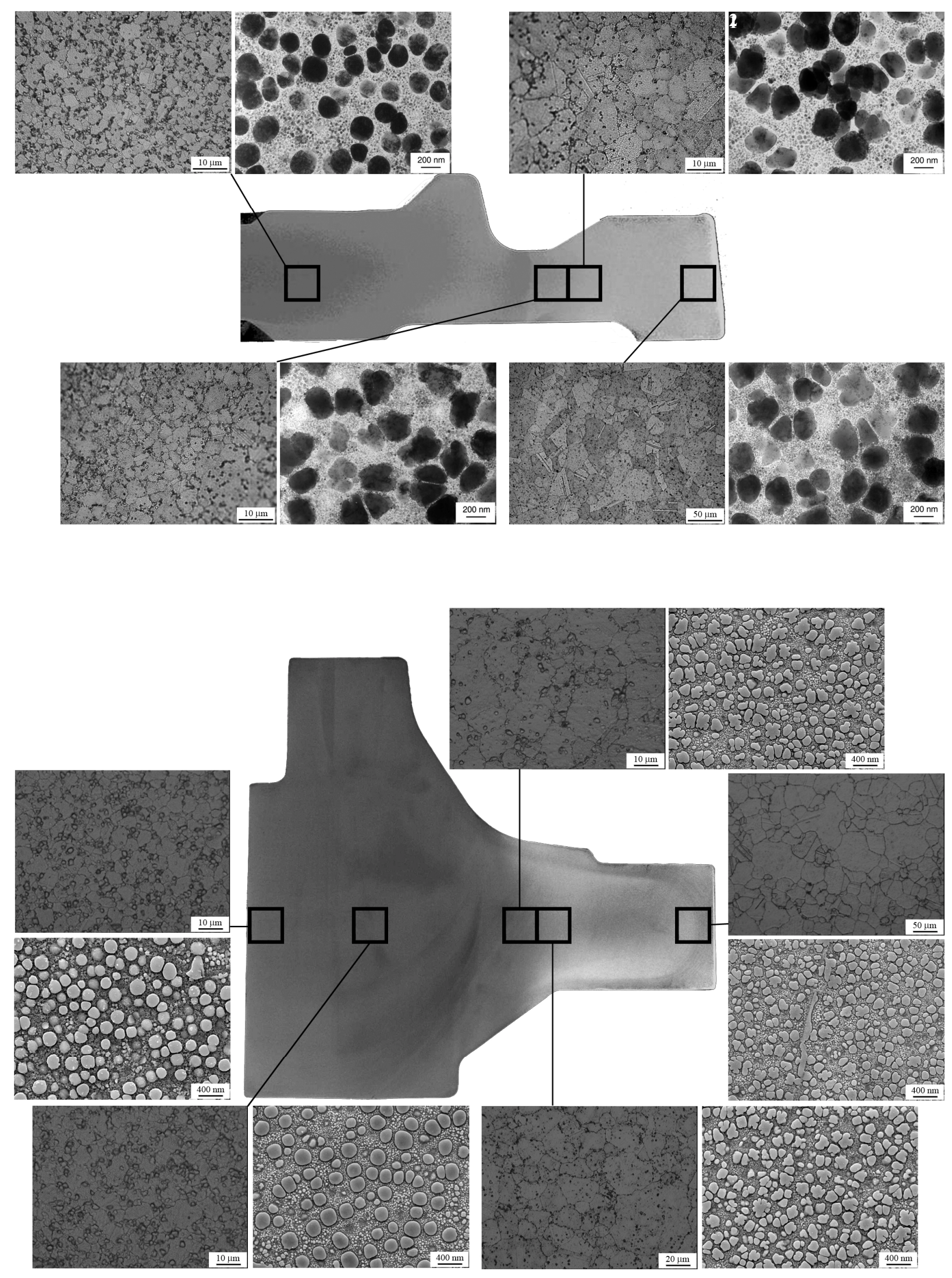

Figure 9 Macro/Micro-Structure of geometries A (top) and B (bottom) produced with a dual-microstructure (fine grain region appears as matt grey in colour, left and coarse grain region appears as lighter grey, right) 
Figure 10 below shows how both the grain size and primary $\gamma^{\prime}$ volume fraction vary as a function of radial position at the midheight of their respective geometries. Component $\mathrm{A}$ is represented by the filled circles, B the open circles. The dotted lines represent the limits of the transition region based on grain size.

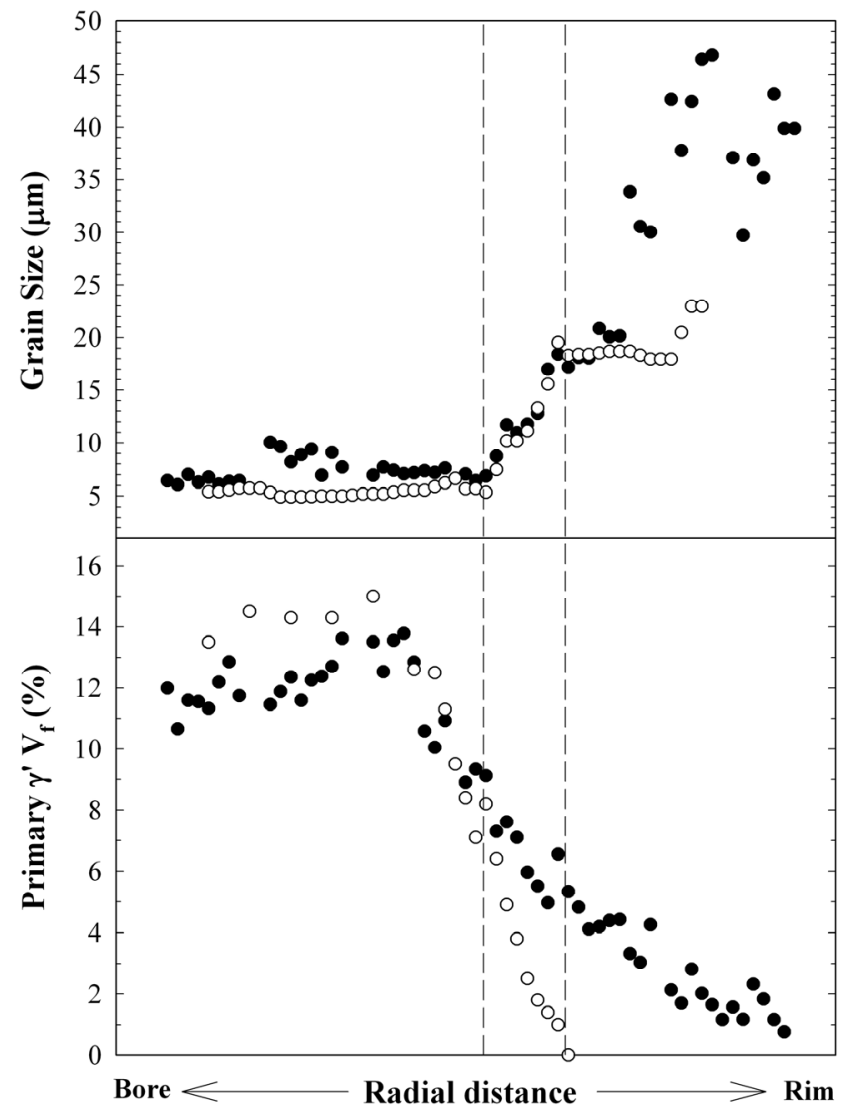

Figure 10 Grain size and Primary $\gamma^{\prime}$ volume fraction as a function of radial distance in components shown in Figure 9.

$($ Closed circles $=\mathrm{A}$, Open circles $=\mathrm{B})$

Apparent from Figure 10 is that irrespective of the differences in component geometry, the transition region width is the same and grain growth is associated with a minimum volume fraction of primary $\gamma^{\prime}$ above which no grain growth occurs. Subtle differences are observed in the coarse grain regions of the two components. The average grain size and volume fraction of primary $\gamma^{\prime}$ are both slightly higher in component A than those measured in component B. These subtle differences have arisen as a direct result of differences in the processing parameters used for these two components, primarily the supersolvus temperature and the total cycle time chosen.

\section{$\underline{\text { Grain Boundary Character }}$}

In addition to obtaining grain size and primary $\gamma^{\prime}$ distribution, grain boundary character was also assessed. The character of grain boundaries in nickel-base superalloys is known to impact upon mechanical properties such as creep and fatigue crack growth [9]. In fine grain structures the presence of primary $\gamma^{\prime}$ upon the grain boundaries effectively prevents any change in the grain boundaries from occurring during either the solution heat treatment or quench. In coarse grain microstructure the increased grain size increases grain boundary length (whilst overall grain boundary area is reduced) and the absence of pinning primary $\gamma^{\prime}$ allows for greater variation in the grain boundaries that develop.

The solution temperature fixes the grain size and the cooling rate from the solution temperature sets the initial $\gamma^{\prime}$ distribution and grain boundary structure. For fast cooling rates the secondary $\gamma^{\prime}$ is small and grain boundaries remain smooth in nature. As the cooling rate decreases, either through design intent or through increase in material bulk (due to geometry) the material is at temperature for longer and the secondary $\gamma^{\prime}$ has time to grow larger. As growth continues the secondary $\gamma^{\prime}$ impinge upon the grain boundaries and cause them to become 'serrated' [10].

Figure 11 below shows typical grain boundaries from fast and slow cooled coarse grain RR1000. The mechanisms behind the formation of grain boundary serrations are fairly well understood although they have not been extensively documented. Even less well documented are the attempts that have been made to characterise the degree of grain boundary serration.

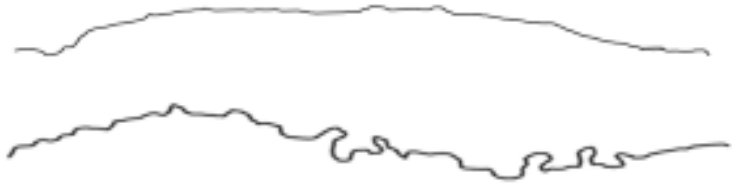

Figure 11 Appearance of 'smooth' (top) and 'serrated' grain boundaries (bottom)

The degree of grain boundary serration can be easily quantified by use of the grain boundary curvature ratio (GBCR). The GBCR formula is shown below, where $l$ is the length of the actual grain boundary and $x$ is the shortest distance between triple points [11].

$$
G B C R=\frac{l}{x}
$$

The GBCR values for component B are shown below, Figure 12 .

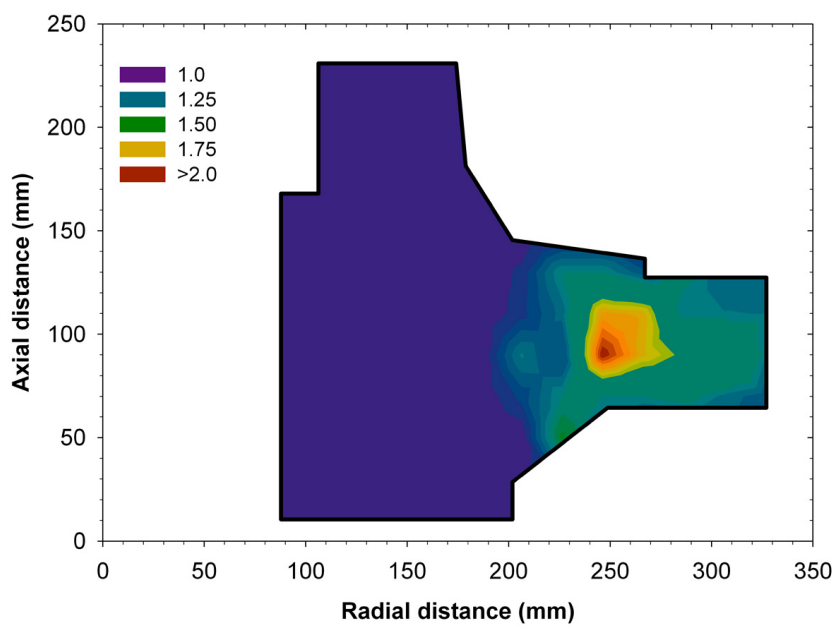

Figure 12 Grain boundary serration as a function of location 
Figure 12 shows that GBCR is greatest where the cooling rate is slowest, i.e. the thickest section, and that the actual grain boundary length is twice that of the shortest distance between the triple points. The GBCR decreases towards the surfaces of the forging, as the cooling rate increases. Understanding microstructural phenomenon such as GBCR and their relationships to process parameters and mechanical properties are the key to successfully designing with dual microstructure components.

\section{$\underline{\text { Secondary and Tertiary } \gamma^{\prime}}$}

Image analysis techniques were also performed for the secondary and tertiary $\gamma^{\prime}$ precipitates in both components. The results for component A have been previously reported [6].

In the previous work on component $\mathrm{A}$ it was noted that both the volume fraction and the secondary $\gamma^{\prime}$ morphology changed markedly, in and approaching, the transition region. Immediately prior to the transition region there is a reduction in the secondary $\gamma^{\prime}$ volume fraction, whilst simultaneously, the secondary $\gamma^{\prime}$ distribution changes from unimodal to bimodal with two distinct population sizes observed.

Information on secondary $\gamma^{\prime}$ size and volume fraction was collected for component $\mathrm{B}$. However, it is not easily presentable within the limitations of a technical paper, and therefore it was decided that rather than looking at the absolute precipitate sizes that it would be better to look at the total number of distributions present in the forging as a function of location.

Figure 13 below shows for each micro-specimen location the number of distinct $\gamma^{\prime}$ populations. For the fine grain regions there is a quad-modal distribution of $\gamma^{\prime}$ consisting of a unimodal intergranular primary $\gamma^{\prime}$ population and a tri-modal distribution of intragranular $\gamma^{\prime}$, i.e. a singular tertiary $\gamma^{\prime}$ distribution $(<50 \mathrm{~nm})$ and a bimodal secondary $\gamma^{\prime}$ distribution $(50-1000 \mathrm{~nm}$, intragranular).

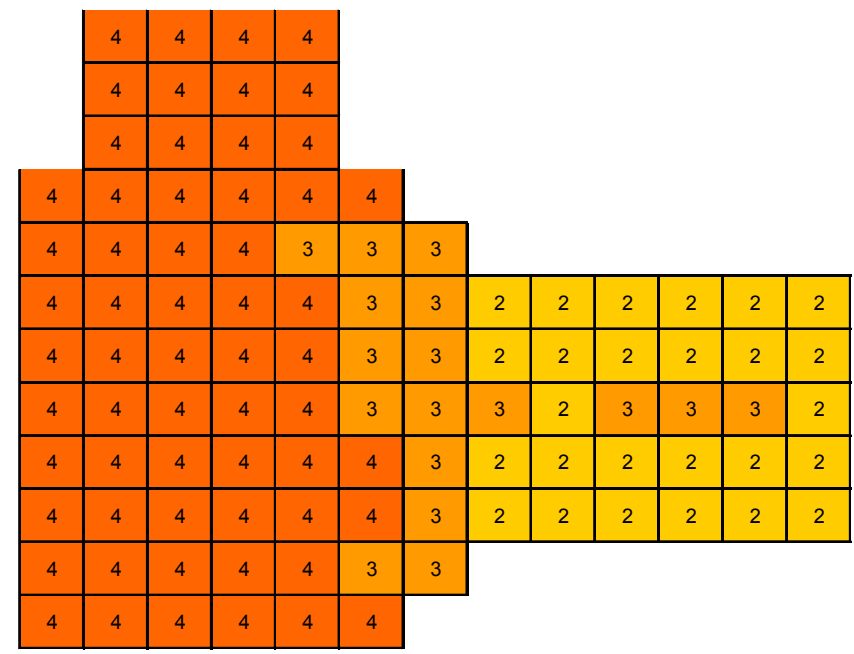

Figure 13 Number of $\gamma^{\prime}$ populations with respect to location

For the majority of the coarse grain locations, and those in the transition region, a bimodal intragranular distribution, i.e. unimodal secondary and tertiary $\gamma^{\prime}$ is present. It should be noted that three of the micro-specimens examined within the coarse grain region exhibited a tri-modal $\gamma^{\prime}$ distribution, due to the presence of a bimodal secondary $\gamma^{\prime}$ formed during the cooling cycle from the DMHT solution cycle, or the subsequent ageing heat treatment.

The other areas of the macro-slice exhibiting a tri-modal $\gamma^{\prime}$ distribution are those coincident with the start of the transition region. From the data presented earlier, Figure 7, we know that primary $\gamma^{\prime}$ is present in this area and examination of the micrographs shows that these areas now exhibit a unimodal secondary $\gamma^{\prime}$ distribution, the larger arm of the bimodal distribution having been removed, suggesting that the secondary $\gamma^{\prime}$ solvus has been exceeded in this region.

Tertiary $\gamma^{\prime}$ measurements were also conducted on both components. It was found that in line with expectation that tertiary $\gamma^{\prime}$ size was largely unaffected by the other microstructural changes and that tertiary $\gamma^{\prime}$ sizes were consistent throughout the entirety of the forging.

\section{Non Destructive Evaluation}

In order to assess the success of the DMHT process at producing a transition zone at a consistent radial location each of the components was inspected using standard ultrasonic inspection techniques. Figure 14 below shows the resultant C-scan plot for component B. The darker blue region (fine grain) and the light blue / green region (coarse grain) are clearly distinguishable. The radial position of the transition region for all of the components produced to date is consistent with the macro-slice investigations and the design intent.
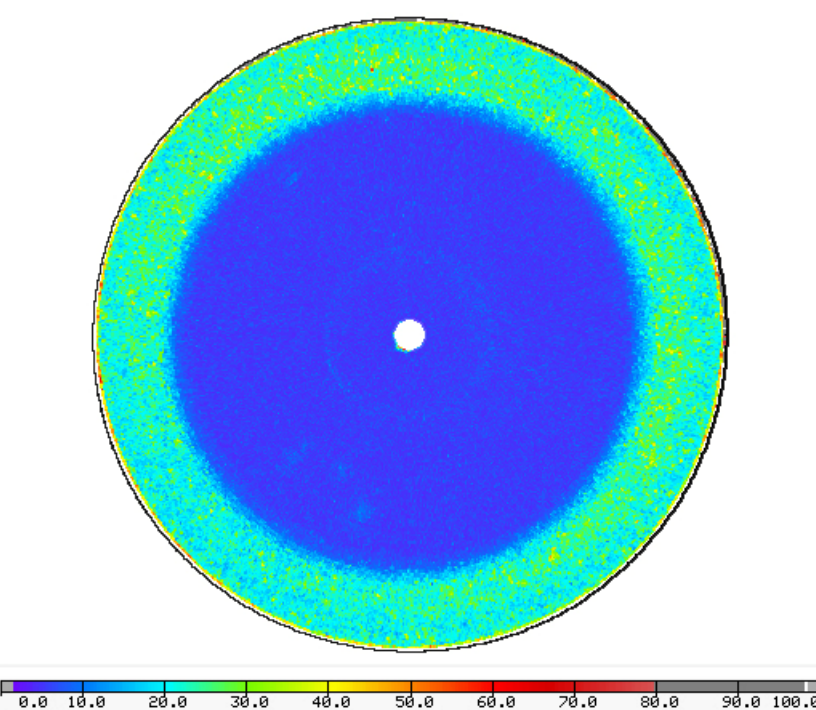

Figure $14 \mathrm{C}$-scan plot of component A showing consistent radial location of transition region 


\section{Mechanical Property Assessment}

Figure 15 below shows the effect of microstructure on the tensile properties and creep life. The increased grain size results in a reduction in the proof strength at this temperature. The values of proof and tensile strength presented here are consistent with earlier work on conventional sub and supersolvus processed RR1000 [12]. The reduction in tensile properties corresponds to an increased time to rupture. For the conditions presented below the coarse grain RR1000 offers a greater than ten fold increase in stress rupture life compared to the fine grain. This is due partly to the increased grain size, but also the greater volume fraction of secondary and tertiary $\gamma^{\prime}$ available from the dissolving primary $\gamma^{\prime}$, leading to improved dislocation creep properties. The scatter in the tensile results is attributed to the specimen cooling rate and hence secondary $\gamma^{\prime}$ size.

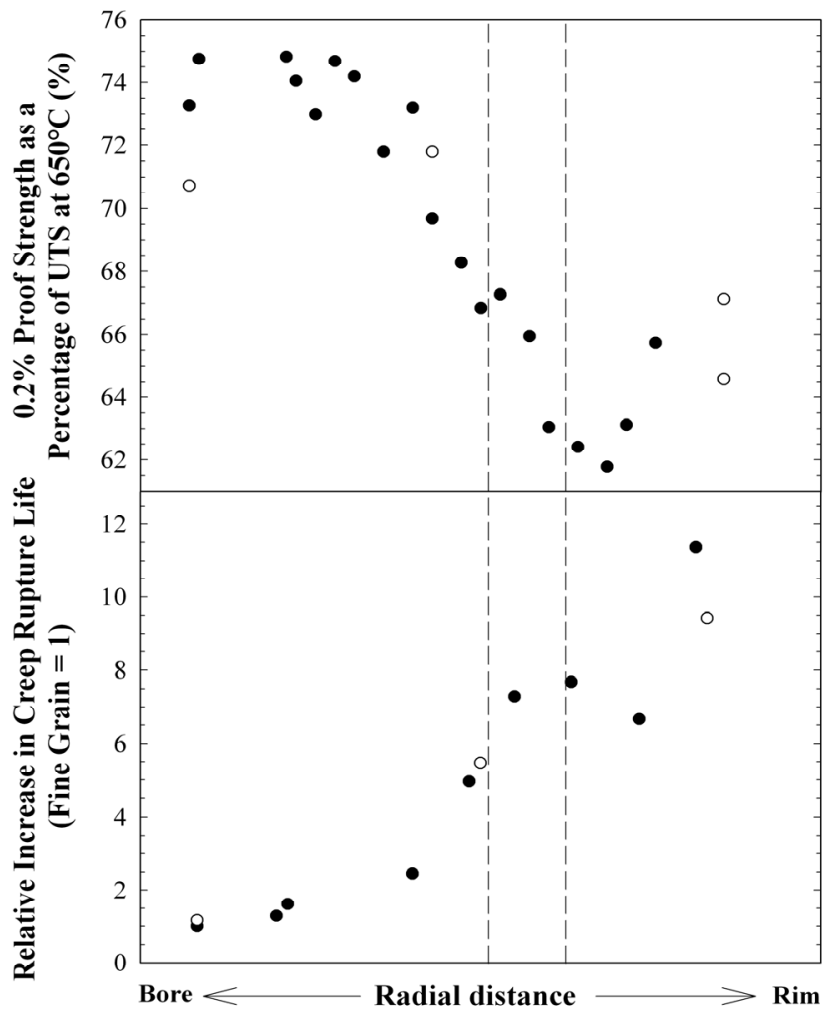

Figure $150.2 \%$ proof strength at $650^{\circ} \mathrm{C}$ as a percentage of UTS and creep rupture life $\left(750^{\circ} \mathrm{C}, 460 \mathrm{MPa}\right)$ as a function of radial distance in dual-microstructure components shown in Figure 9.

Figure 16 shows the effect of microstructure on the minimum creep strain rate at a range of creep test conditions. The plot shows that minimum creep strain rates in the bore and transition microstructures are very similar and are significantly higher than those witnessed in the rim microstructure. It should be noted at this point that the transition region creep specimens were orientated such that the gauge length encompassed the radially variable grain size and that the samples were found to preferentially fail towards the fine grain end, coincident with the observed creep response.

A further series of creep tests $\left(750^{\circ} \mathrm{C}, 460 \mathrm{MPa}\right)$ were performed on samples cut from different radial locations within component A. These samples had the gauge length orientated parallel to the hoop direction. Transmission electron microscopy was performed on the failed creep samples to evaluate the interaction of dislocations with the various sizes and distributions of $\gamma^{\prime}$ precipitates.

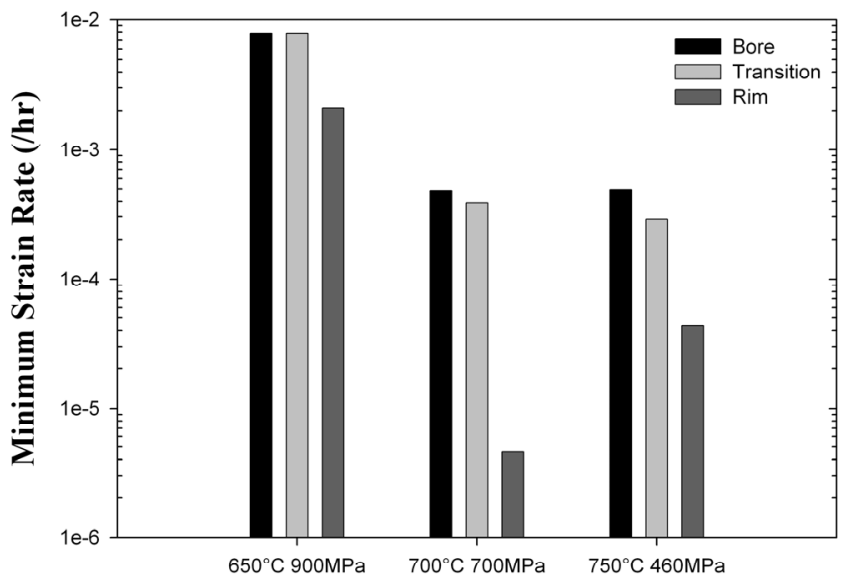

Creep Test Conditions

Figure 16 Effect of microstructure on minimum strain rate for a number of creep test conditions

Figure 17 presents two images from two differently oriented grains in the same creep strain sample located in a mid-rim position. The deformation seen is highly dependent on grain orientation: Figure 17(a) shows the dislocations predominantly looping around the secondary precipitates but also evidence of planar deformation and cutting of the precipitates with the resultant stacking faults, 17(b) shows deformation twinning in another grain.
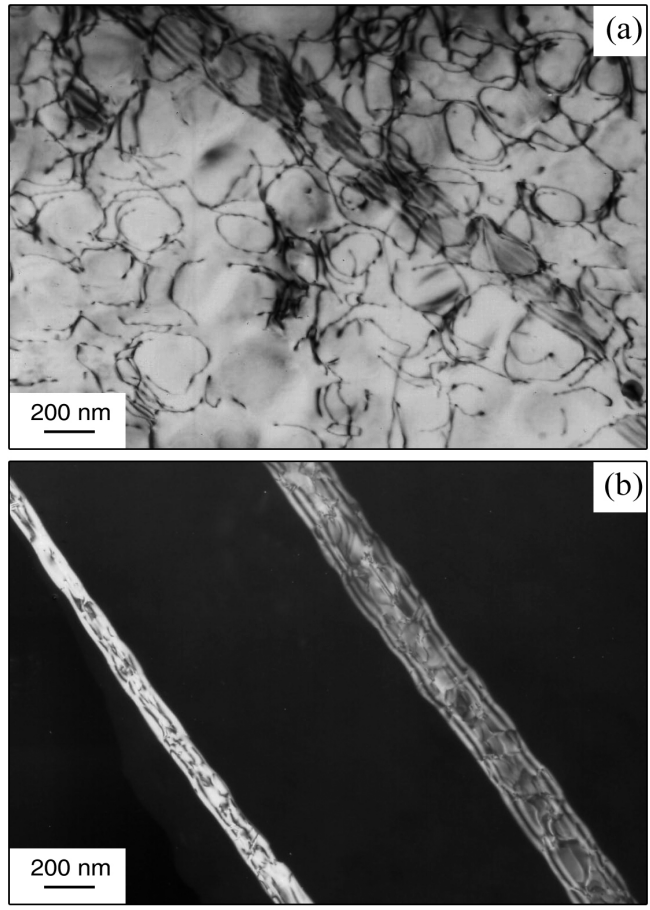

Figure 17 Transmission Electron Microscope images showing interactions of dislocations with $\gamma^{\prime}$ precipitates in different grain orientations in a dual microstructure component. 
Similar micro-mechanisms of creep deformation were observed throughout the disc in both coarse and fine grain regions. A greater variation occurred between grains of different orientation rather than with radial position. Hence the observed differences in minimum creep rate are attributable principally to an increase in secondary and tertiary $\gamma^{\prime}$ volume fraction; rupture life is also affected by the grain size. No significant coarsening of the secondary precipitates was noted.

Fatigue tests were conducted on specimens from each different microstructural region using a trapezoidal waveform with a stress ratio of zero at a range of temperatures. Figure 18 below shows the effect of microstructure on fatigue endurance at $300^{\circ} \mathrm{C}$ and $700^{\circ} \mathrm{C}$ for identical test conditions. The low cycle fatigue data show longer endurance lives at $300^{\circ} \mathrm{C}$ compared to $700^{\circ} \mathrm{C}$. However, it is noticeable that the low cycle fatigue endurance at $700^{\circ} \mathrm{C}$ remains consistent irrespective of microstructure. The high cycle fatigue results show lower fatigue endurance at $300^{\circ} \mathrm{C}$ compared to $700^{\circ} \mathrm{C}$ although endurance at both temperatures decreases with increasing grain size.

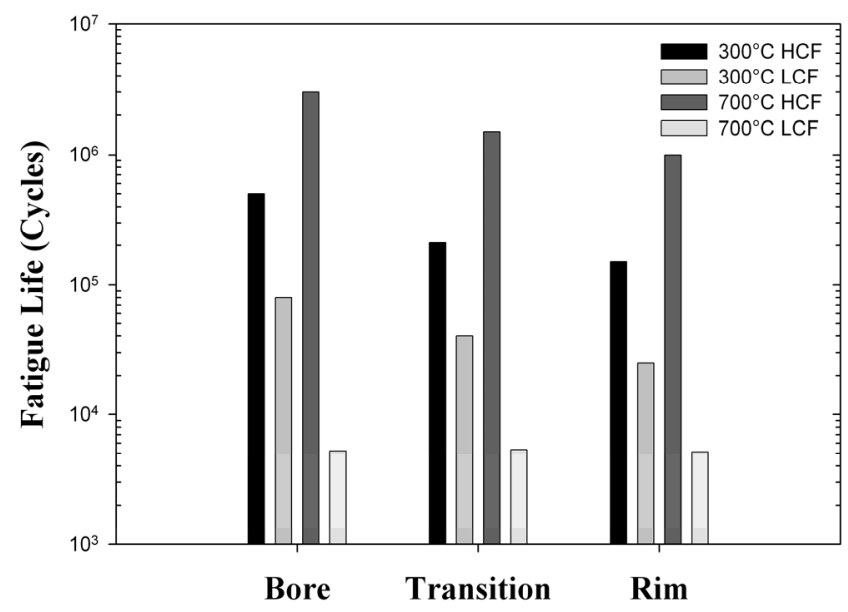

Figure 18 Low and High Cycle Fatigue lives as a function of microstructure and test temperature

It was found in that the coarse grain microstructure exhibited lower fatigue lives at $300^{\circ} \mathrm{C}$ due to crack initiation at crystallographic features, Figure 19.

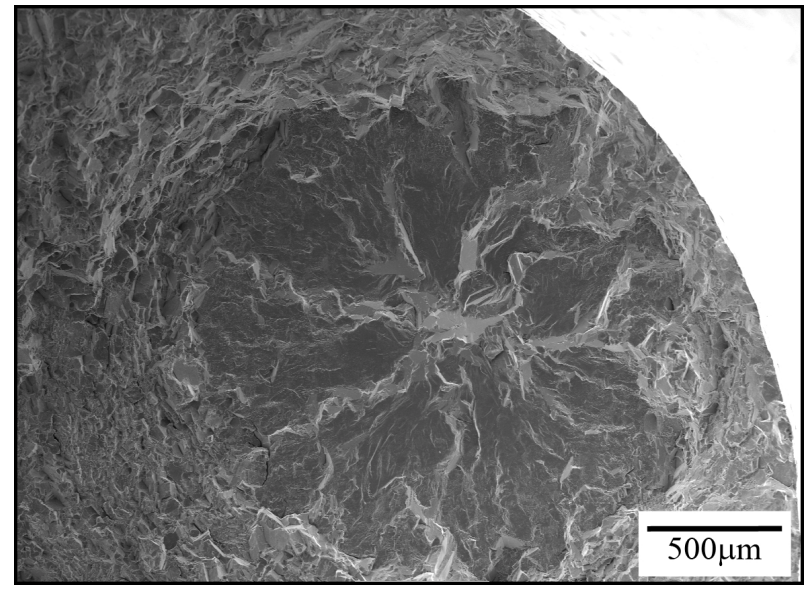

Figure 19 Fatigue crack initiation site in a coarse grain high cycle fatigue specimen tested at $300^{\circ} \mathrm{C}$
Fatigue crack growth (FCG) testing was conducted using a corner crack specimen geometry at a range of temperatures under either a baseline, 1-1-1-1 trapezoidal cycle or with $120 \mathrm{~s}$ dwell at peak load. Figure 20 shows the effect of microstructure and dwell time on fatigue crack growth rates in dual-microstructure RR1000 at $700^{\circ} \mathrm{C}$.

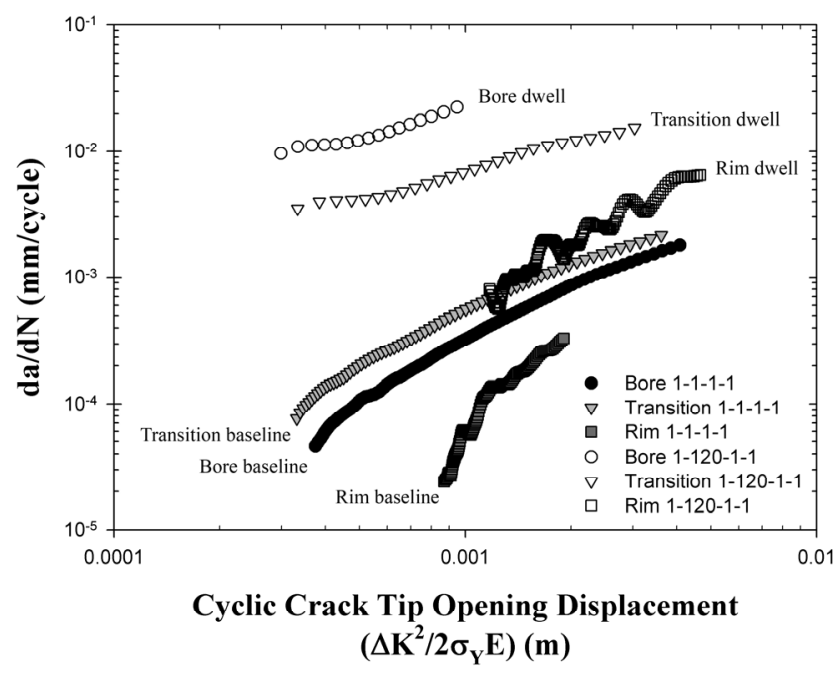

Figure 20 Fatigue Crack Growth rates at $700^{\circ} \mathrm{C}$ when tested under baseline and dwell conditions as a function of cyclic crack tip opening displacement

Fatigue crack growth response for baseline cycles for bore and transition region microstructures is very similar within the level of scatter associated with FCG testing. The baseline rim microstructure exhibits the slowest FCG rate. The imposition of a $120 \mathrm{~s}$ dwell increases the fatigue crack growth rate by approximately two orders of magnitude in the bore (fine grain) microstructure. The same dwell cycle for the coarse grain rim specimen only increases the fatigue crack growth rate by approximately one order of magnitude. The coarse grain 120s dwell fatigue crack growth rate is considered equivalent to the baseline growth rate at this temperature. The transition region specimens tested at this temperature show baseline crack growth rates equivalent to the fine grain microstructure within scatter. The 120s dwell at peak load results in a crack growth rate between those observed for the fine and coarse grain microstructures.

Examination of the fracture surfaces reveals transgranular crack growth for all microstructures under baseline loading conditions. The application of the 120 s dwell at peak load changes the crack growth mechanism to intergranular for all of the microstructural regions.

Notch fatigue testing was conducted on a specimen with a stress concentration factor $\left(\mathrm{K}_{\mathrm{t}}\right)$ of approximately two. Figure 21 below shows the effect of microstructure on notch fatigue endurance at $750^{\circ} \mathrm{C}$ for a $1-1-1-1$ and a $1-120-1-1$ cycle, relative to typical behaviour of the bore microstructure under the baseline cycle, which for the applied stress level returned a life of $\sim 10,000$ cycles. 


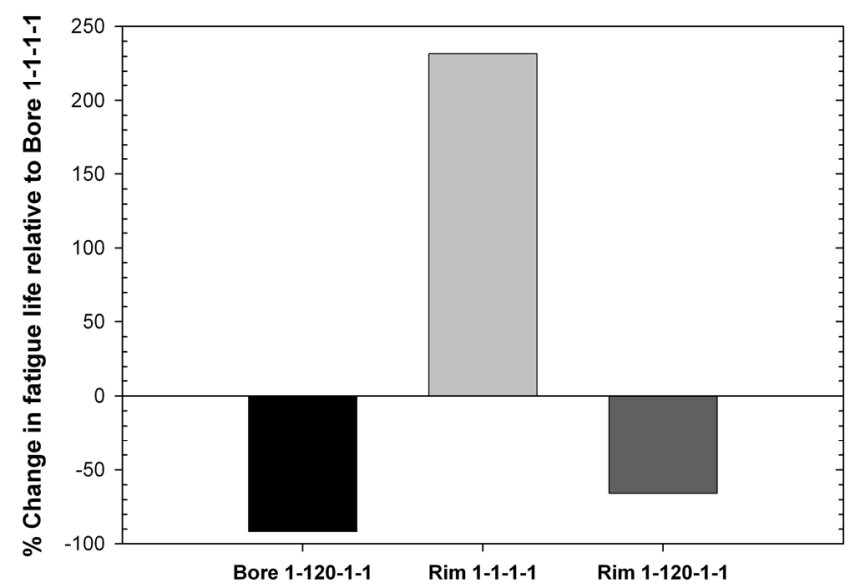

Figure 21 Effect of microstructure and dwell on notch fatigue endurance at $750^{\circ} \mathrm{C}$ at a given stress value

Applying a 120 s dwell at peak load results in a $90 \%$ reduction in fatigue life for the bore microstructure, relative to the baseline cycle. However, at this temperature and for this notch feature the coarse grain rim specimen under a baseline loading cycle exhibits an increase in fatigue endurance of over $200 \%$ over the fine grain. This coarse grain fatigue benefit is also witnessed after the application of a $120 \mathrm{~s}$ dwell at peak load, where the coarse grain shows a $60 \%$ debit relative to the fine grain baseline, compared to the $90 \%$ witnessed in the fine grain sample.

\section{Summary \& Conclusions}

Rolls-Royce has successfully demonstrated the capability to produce dual microstructure components in a range of turbine disc forging geometries. Ladish have successfully developed and validated a process modeling approach based on the understanding of the key process variables to deliver these components in an acceptable time frame and without the need for multiple, time-consuming thermocouple trials.

A number of parts have been produced with excellent part-to-part conformity. Highly detailed microstructural assessment has been performed on a number of component geometries and the results observed linked back to thermal and thermo-mechanical process history. The process developed met the design intent in terms of grain size requirements and location and further understanding of the precipitation phases was gained through extensive microstructural analysis.

Mechanical testing has been conducted to confirm the perceived benefits / limitations of the DMHT solution. High proof strengths and fatigue lives were observed in the bore, whilst the rim region displayed greatly improved creep properties and resistance to crack growth, in particular under dwell conditions. Notched fatigue testing has shown that under certain conditions coarse grain microstructures may out perform fine grain under both baseline and dwell conditions.

In summary Rolls-Royce has developed a production capable process for the manufacture of dual-microstructure components, with a range of geometries produced encompassing those applications likely to require this technological solution.

\section{Acknowledgements}

The authors would like to express their sincere thanks to Dr Mark Hardy of Rolls-Royce plc., Professor Paul Bowen of University of Birmingham and Professor Martin Bache of Swansea University for many useful discussions and in help in preparing this manuscript. The authors also acknowledge the contributions of Dr Howard Stone and Dr Cathie Rae of the University of Cambridge.

Part of this research has been undertaken in the MACE (Materials for Arduous Cycle and Emissions) Project (TP/2/ET/6/I/10037), which forms part of the Department of Trade and Industry Technology Programme.

\section{References}

1. J. Gayda, T.P. Gabb and P.T. Kantzos "eat Treatment Devices and Method of Operation Thereof to Produce Dual Microstructure Superalloy Disks", US Patent 6,660,110, December, 2003

2. J. Gayda et al. "Heat Treatment Technology for Production of Dual Microstructure Superalloy Disks", NASA TM 2002211558, April 2002

3. D.P. Mourer and J.L. Williams "Dual Heat Treat Process Development for Advanced Disk Applications", pp401-408, Superalloys 2004, Warrendale, PA

4. G.F. Mathey "Method of making superalloy turbine disks having graded coarse and fine grains", US Patent 5,312,497, May 17, 1994

5. M.C. Hardy et al. "Developing Damage Tolerance and Creep Resistance in a High Strength Nickel Alloy for Disc Applications", pp83-90, Superalloys 2004, Warrendale, PA

6. L.D. Connor et al. "Dual Microstructure Heat Treated Discs: Microstructural Characterisation" pp321-332, Parsons 2007 Power Generation in an Era of Climate Change, Glasgow, UK

7. ASTM E112-96 "Standard Test Methods for Determining Average Grain Size", 2004

8. M.D. Abramoff, P.J. Magelhaes and S.J. Ram "Image Processing with ImageJ", Biophotonics International, Volume 11, Issue 7, pp36-42, 2004

9. J. Telesman et al. "Microstructural variables controlling timedependant crack growth in a P/M superalloy", pp215-224, Superalloys 2004, Warrendale, PA

10. R.J. Mitchell, H.Y. Li and Z.W. Huang "On the formation of serrated grain boundaries and fan-type structures in an advanced polycrystalline nickel-base superalloy" Journal of Materials Processing Technology, 2008, To be published

11. T.P. Gabb, A. Garg, D.L. Ellis and K.M. O'Connor "Detailed microstructural characterization of the disk alloy ME3", NASA/TM-2004-213066, 2004

12. A. Banik et al. "Low cost powder turbine components", pp571-576, Superalloys 2004, Warrendale, PA 